\title{
Sphenoid Sinus Mucocele Caused by a Completely Thrombosed Intracavernous Carotid Artery Aneurysm: An Unusual Association
}

\author{
Alessandro Villa ${ }^{1}$ Michelangelo De Angelis ${ }^{1}$ Ivan Piscevic ${ }^{2}$ Domenico Solari ${ }^{1}$ Luigi Maria Cavallo ${ }^{1}$ \\ Paolo Cappabianca ${ }^{1}$ \\ ${ }^{1}$ Division of Neurosurgery, Department of Neurosciences and \\ Reproductive and Odontostomatological Sciences, Università degli \\ Studi di Napoli Federico II, Naples, Italy \\ ${ }^{2}$ Clinical Centre of Serbia, Clinic of Neurosurgery, Belgrade, Serbia \\ Address for correspondence Alessandro Villa, MD, Division of \\ Neurosurgery, Department of Neurosciences and Reproductive and \\ Odontostomatological Sciences, Università degli Studi di Napoli \\ Federico II, Via S. Pansini 5, Napoli 80131, Italy \\ (e-mail: alevilla83@libero.it).
}

J Neurol Surg Rep 2014;75:e108-e112.

\begin{abstract}
Background Mucocele is an inflammatory disease caused by the retention of mucoid secretions within a paranasal sinus. Although rare, the presence of a vascular lesion inside the sphenoid sinus could determine ostium obstruction, thus causing mucocele development.

Clinical Presentation An 84-year-old woman was referred to our institution due to a lesion abutting into the sphenoid sinus; she was complaining of constrictive frontal headache, progressive worsening of visual acuity in the left eye; later, sudden homolateral ptosis and diplopia occurred. The radiologic evidence was consistent with the

Keywords

- sphenoidal mucocele

- aneurysm

- endoscopic endonasal surgery

- skull base surgery

- sinus surgery diagnosis of thrombosed aneurysm of the right intracavernous carotid artery, surrounded by sphenoidal mucocele. The patient underwent an endoscopic endonasal transsphenoidal approach exclusively for sphenoid mucocele drainage.

Conclusion Although rare, the presence of a vascular lesion inside the sphenoid sinus has to be considered among the possible diagnostic hypotheses of masses abutting in this cavity; the association with mucocele is even more rare and, to date, has not been described.
\end{abstract}

\section{Introduction}

Mucocele is an inflammatory disease caused by the retention of mucoid secretions within a paranasal sinus; the progressive volume expansion of this lesion can lead to thinning and/ or erosion of the bony walls of the sinus. According to the pathogenetic mechanism, mucocele can be considered primary, developing as a retention cyst of degenerated epithelial mucous glands, or secondary, as a result of sinus ostium obstruction or cystic degeneration of inflammatory polyps. Frontal sinus mucoceles are the most common, whereas those involving the sphenoid sinus are more unusual, accounting for 1 to $2 \%$ of all mucoceles. Although rare, the presence of a vascular lesion inside the sphenoid sinus may determine ostium obstruction, thus causing mucocele development. Hence it should be considered among the possible diagnostic hypotheses of masses abutting inside the sphenoid sinus.

\section{Case Report}

An 84-year-old woman with history of hypertension, noninsulin-dependent diabetes, and bilateral cataract, operated on in both eyes, was referred to our institution due to a lesion abutting into the sphenoid sinus. Three months before hospital admittance she started complaining of a constrictive frontal headache, progressive worsening of visual acuity in received

August 30, 2013

accepted after revision

February 20, 2014

published online

May 2, 2014
DOI http://dx.doi.org/ 10.1055/s-0034-1373666. ISSN 2193-6358. (c) 2014 Georg Thieme Verlag KG
Stuttgart · New York

License terms

(c) $(1) \$$ 
the left eye; later, sudden homolateral ptosis and diplopia occurred.

A postcontrast head computed tomography scan revealed a large nonenhancing lesion within the sphenoid sinus, expanding and remodeling both its medial walls, consistent with the diagnosis of sphenoidal mucocele. At the center of the lesion, a low-density round mass, outlined by a hyperdense rim, suggestive of calcium deposits, was

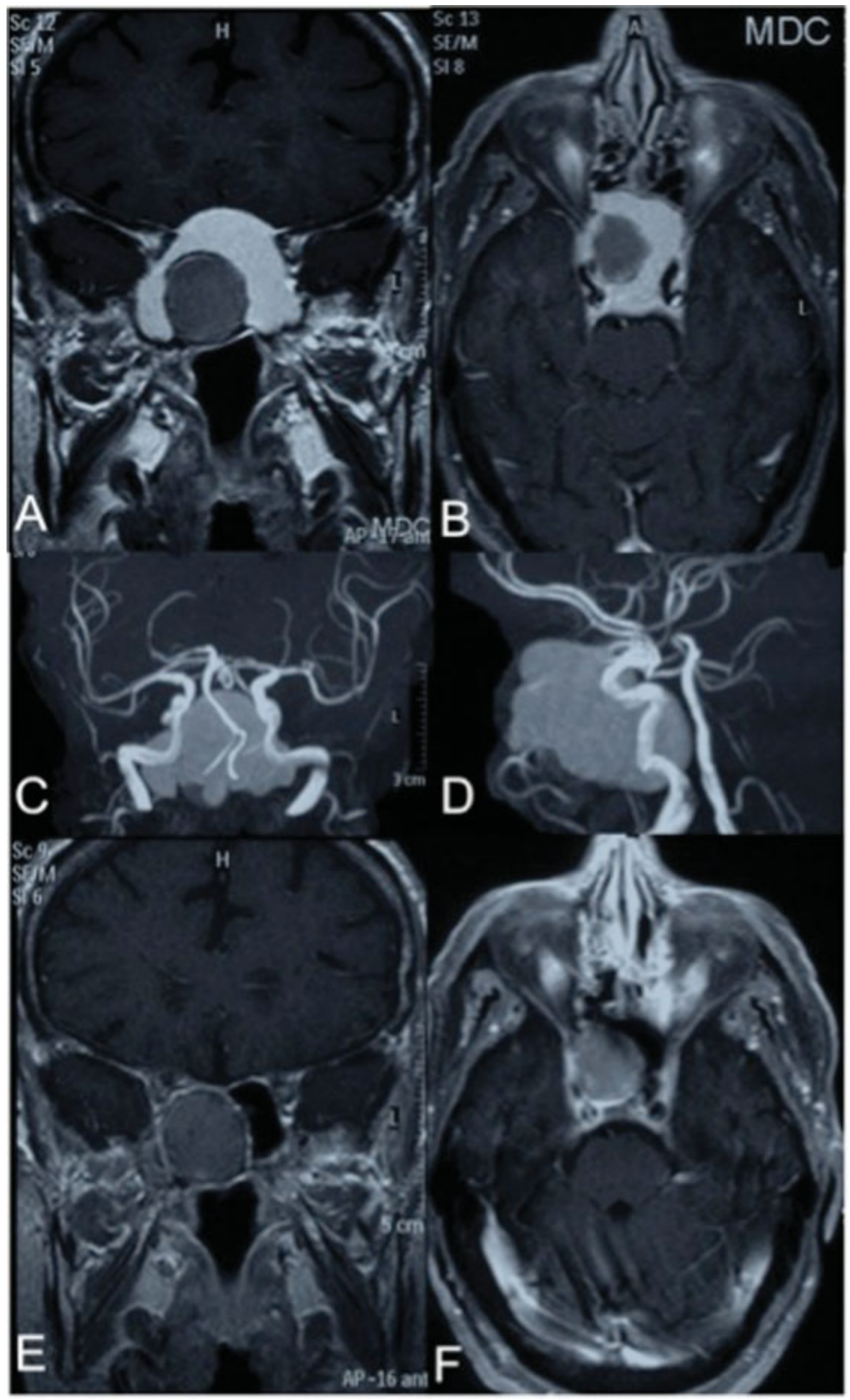

Fig. 1 Preoperative postcontrast magnetic resonance imaging (MRI). (A) Coronal and (B) axial scans showing a homogeneous, spontaneously hyperintense, nonenhancing after contrast injection mucocele filling the sphenoid sinus cavity. The central round mass has a heterogeneous signal with a hypointense rim, consistent with the suspicion of a thrombosed aneurysm of the right intracavernous carotid artery. (C, D) Angiographic sequences showing signal void within the aneurysm. (E, F) Postoperative MRI showing the complete removal of the mucocele and the unchanged thrombosed aneurysm of the right intracavernous carotid artery. 
identified; areas of calcification were also observed inside the lesion. A brain magnetic resonance imaging (MRI), integrated also by angiographic sequences, confirmed the presence of a homogeneous round mass inside the sphenoid sinus cavity, nonenhancing after intravenous gadolinium injection, markedly and spontaneously hyperintense both in $\mathrm{T} 1$ and $\mathrm{T} 2$. The second inner lesion appeared heterogeneous and lined by a thin hypointense rim. The angiographic study showed no flow within this lesion, and, similarly, no flow alteration within the distal vessels of the intracerebral circulation was noted. With regard to these features, suspicion of thrombosed aneurysm of the right intracavernous carotid artery, surrounded by sphenoidal mucocele, was indicated (-Fig. 1A-D).

Upon admission to our department, the patient's neurologic examination showed bilateral amaurosis (patient was already blind in the right eye) and a complete left ophthalmoplegia. The physical examination was unremarkable.

The patient underwent an endoscopic endonasal transsphenoidal approach for sphenoidal mucocele drainage. After complete mucocele removal, the aneurysm was clearly identified as a mass arising from the right wall of the sphenoid sinus, occupying mostly the right half of the sphenoid sinus cavity. It was confirmed that the aneurysm was thrombosed. It appeared to be solid, covered by respiratory mucosa, and not pulsating (-Fig. 2A, B). In the upper counterpart of the sphenoid sinus, the transmitted pulsation of the uncovered left internal carotid artery, protruding inside the sphenoid cavity at the level of the planum sphenoidale, was observed.
The mucosa inside the sphenoid sinus appeared normotrophic and normochromic, and no signs or symptoms of systemic inflammation were disclosed preoperatively. As such, we did not suspect any mucosal infection and no culture examination was performed. Antibiotic irrigation of the sphenoid cavity was ultimately performed.

As already discussed with the patient, according to clinical conditions and radiologic features, no surgical or endovascular treatment of the aneurysm was attempted. The patient had an uneventful postoperative course and was discharged on postoperative day 1 .

A 3-month postoperative MRI confirmed the removal of the sphenoid mucocele, although the aneurysm had not changed (-Fig. 1E, F).

At the 6-month follow-up examination, the patient was relieved from headaches, but bilateral amaurosis and complete ophthalmoplegia in the left eye had not changed. At this time, the endoscopic endonasal control showed complete healing of the mucosa inside the sphenoid sinus, with normally pinkish mucosa covering the aneurysm mass inside it (-Fig. 2C, D).

\section{Discussion}

Sphenoid mucoceles are rare lesions ${ }^{1,2}$ representing only 1 to $2 \%$ of all sinus mucoceles. ${ }^{1,3-5}$ According to the pathogenetic mechanism, mucocele can be primary, when developed as retention cysts of the sinus epithelium mucous glands, and secondary, caused by sinus ostium obstruction, eventually

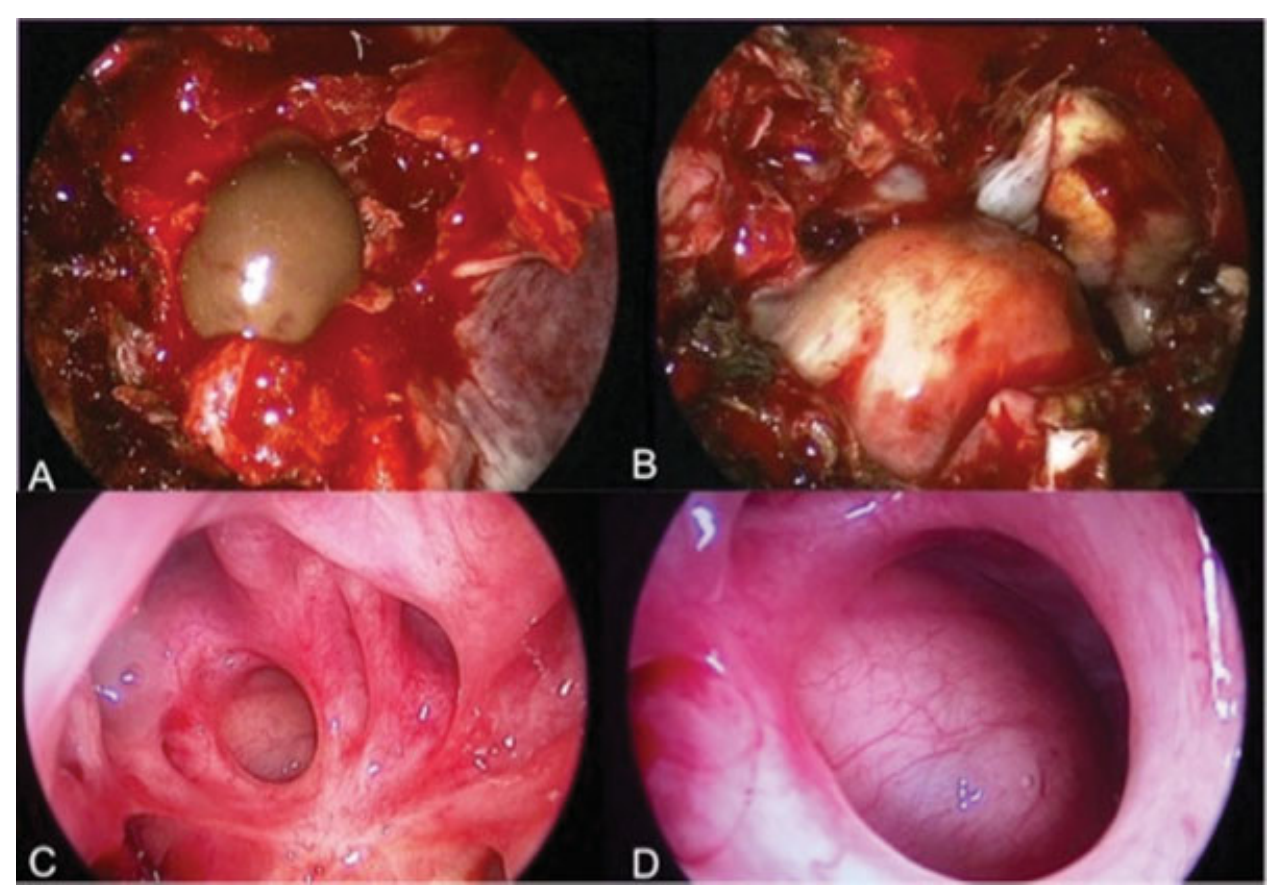

Fig. 2 Intraoperative images of the endoscopic endonasal transsphenoidal approach. (A) Initial drainage of the mucocele after the opening of the anterior wall of the sphenoid sinus. (B) After the complete drainage of the mucocele, the thrombosed aneurysm covered by normal respiratory sinus mucosa is seen in the right part of the sphenoid sinus cavity. The mucocele-eroded bony walls of the sphenoid sinus uncovered the dura of the planum sphenoidale and the left carotid artery in its paraclinoidal tract. (C) Images of the outpatient clinic endoscopic control at 6-month follow-up showing the normal pinkish and trophic mucosa of the sphenoid sinus. (D) A close-up view of the thrombosed aneurysm. 
from masses extending inside the sinus cavities. ${ }^{6}$ This latter condition corresponds to our case.

The increase in volume of the mucocele can lead to erosion and thinning of the bony walls of the sinus. Sphenoid sinus mucoceles may present with different symptoms due to compression and/or injury of the surrounding neurovascular structures such as cranial nerves II, III, IV, and VI, the carotid arteries, the cavernous sinuses, and the pituitary gland. The symptoms related to cranial nerve involvement ( $50 \%$ of cases) often lead to the diagnosis and may require timely surgery of decompression. Defects of eye motility occur in $~ 30$ to $50 \%$ of cases of sphenoid mucoceles with the oculomotor nerve affected more frequently than the abducent and the trochlear nerves. ${ }^{1,3,7,8}$ Visual acuity worsening is mostly progressive, but sudden blindness may occur. ${ }^{2-4,9,10}$ However, the most common symptom is headache, typically supraorbital or retroorbital, occurring in 70 to $80 \%$ of cases. ${ }^{3,11,12}$ In our case, the mucocele extended in an anterosuperior direction inside the sphenoid sinus, in toward the superior orbital fissure. We could assess that signs and symptoms correlated mostly with the mucocele compressing the left cavernous sinus. The headache ceased after surgery, whereas left ptosis and diplopia remained stable, related to the long-lasting compression of the sphenoidal sinus left medial wall. Concerning the amaurosis, the role of previous cataract surgery should not be underestimated.

Among surgical options for sphenoidal mucocele treatment, transfacial or transcranial approaches have been replaced by the endoscopic endonasal approach. This latter technique is safe and effective, providing a good outcome in terms of sinus opening and mucocele drainage. ${ }^{1,2,4,5,7,13-15}$ Early surgery is strongly recommended in the attempt to achieve cranial nerve function recovery. Although visual loss is often irreversible, ${ }^{7}$ a few cases of postsurgical improvement have been reported. ${ }^{15,16}$

Whereas aneurysms of the intracavernous tract of the internal carotid artery are frequently characterized by partial luminal thrombosis (70\% of cases), aneurysmal lumen complete occlusion is very rare. ${ }^{17}$ These aneurysms often present with third, fifth, or sixth cranial nerve palsies; rarely, they bleed and cause subarachnoid hemorrhage. ${ }^{18}$

The treatment of thrombosed aneurysms is still a challenge, and some authors suggest surgery in symptomatic patients to relieve the mass effect. Lawton et al ${ }^{19}$ made a morphologic and therapeutic classification of thrombosed intracranial aneurysms, proposing that only totally thrombosed aneurysms (type 4) can be treated with watchful waiting. In our case no flow was detected at the angio-MRI within the aneurysm. Thus it was considered a totally thrombosed and, accordingly, conservatively treatable lesion. Similarly, at follow-up we preferred to adopt a conservative approach, evaluating the possible aneurysm reperfusion by means of an MRI integrated by angiographic sequences rather than angiography.

We must emphasize that the endoscopic endonasal approach allows a better view of the pathology and anatomy of the cranial base region. In our case, it permitted us to identify the consistency of the carotid thrombosed aneurysm and to recognize its origin from the cavernous sinus.

The present case report showed an unusual association of thrombosed aneurysm of internal carotid artery and mucocele within the sphenoid sinus, causing severe neurologic symptoms.

The possible presence of a vascular lesion of the internal carotid artery inside the cavernous sinus can be responsible for the development of secondary mucocele and therefore be considered among the different diagnostic hypotheses of space-occupying masses inside the sphenoid sinus cavity.

\section{References}

1 Kataria R, Gupta S, Chopra S, Bagaria H, Sinha VD. Mucocele of the sphenoid sinus: a rare cause of reversible 3(rd) nerve palsy. Ann Indian Acad Neurol 2012;15(2):158-160

2 Hejazi N, Witzmann A, Hassler W. Ocular manifestations of sphenoid mucoceles: clinical features and neurosurgical management of three cases and review of the literature. Surg Neurol 2001; 56(5):338-343

3 Roufail E, Briggs R, Tress B, Kaye AH. Sphenoid sinus mucocele presenting as complete ophthalmoplegia. J Clin Neurosci 2001; 8(4):372-374

4 Darouassi Y, Righini CA, Reyt E. Mucoceles of the sphenoidal sinus: a report of four cases and review of the literature. B-ENT 2005; 1(4):181-185

5 Soon SR, Lim CM, Singh H, Sethi DS. Sphenoid sinus mucocele: 10 cases and literature review. J Laryngol Otol 2010;124(1):44-47

6 Friedman A, Batra PS, Fakhri S, Citardi MJ, Lanza DC. Isolated sphenoid sinus disease: etiology and management. Otolaryngol Head Neck Surg 2005;133(4):544-550

7 Vaphiades MS, Roberson GH. Sphenoid sinus mucocele presenting as a third cranial nerve palsy. J Neuroophthalmol 2005;25(4): 293-294

8 Friedmann G, Harrison S. Mucocoele of the sphenoidal sinus as a cause of recurrent oculomotor nerve palsy. J Neurol Neurosurg Psychiatry 1970;33(2):172-179

9 Casteels I, De Loof E, Brock P, et al. Sudden blindness in a child: presenting symptom of a sphenoid sinus mucocele. $\mathrm{Br} \mathrm{J}$ Ophthalmol 1992;76(8):502-504

10 Wurster CF, Levine TM, Sisson GA. Mucocele of the sphenoid sinus causing sudden onset of blindness. Otolaryngol Head Neck Surg 1986;94(2):257-259

11 Nugent GR, Sprinkle P, Bloor BM. Sphenoid sinus mucoceles. J Neurosurg 1970;32(4):443-451

12 Razmpa E, Naghibzadeh B, Bagheri A, Sadeghi M, Khak M. The clinical manifestation, evaluation and surgical management of sphenoid sinus mucoceles: a case series and literature review. B-ENT 2011;7(2):87-90

13 Giovannetti F, Filiaci F, Ramieri V, Ungari C. Isolated sphenoid sinus mucocele: etiology and management. J Craniofac Surg 2008;19(5): 1381-1384

14 Jolly K, Krishnasamy S, Buch VH, Buch HN, Mathews J. Sphenoid mucocele: an uncommon complication of a rare condition. Scott Med J 2012;57(4):247

15 Haloi AK, Ditchfield M, Maixner W. Mucocele of the sphenoid sinus. Pediatr Radiol 2006;36(9):987-990

16 Bahadir O, Arslan S, Arslan E, Türk A, Ural A. Sphenoid sinus mucocele presenting with unilateral visual loss: a case report. BENT 2011;7(1):65-68

17 Tamburrini G, Puca A. Complete spontaneous thrombosis of a giant aneurysm of the intracavernous carotid artery. Ital J Neurol Sci 1997;18(2):125-126 
e112 Sphenoidal Mucocele and Cavernous Carotid Aneurysm

18 Kurokawa R, Kuroshima Y, Yoshida K, Kawase T. Spontaneous thrombosis of intracavernous internal carotid artery aneurysm and parent artery occlusion in patients with positive balloon test occlusion-two case reports. Neurol Med Chir (Tokyo) 2001;41(9): $436-441$
19 Lawton MT, Quiñones-Hinojosa A, Chang EF, Yu T. Thrombotic intracranial aneurysms: classification scheme and management strategies in 68 patients. Neurosurgery 2005;56(3):441-454; discussion $441-454$ 